\title{
Thickness-Dependent Strain Effect on the Deformation of the Graphene-Encapsulated Au Nanoparticles
}

\author{
Shuangli Ye, ${ }^{1}$ Honghua Huang, ${ }^{1,2}$ Cailei Yuan, ${ }^{2}$ Feng Liu, ${ }^{1}$ Min Zhai, ${ }^{1}$ Xinzhi Shi, ${ }^{1}$ \\ Chang Qi, ${ }^{1}$ and Gaofeng Wang ${ }^{1}$ \\ ${ }^{1}$ Institute of Microelectronics and Information Technology, Wuhan University, Wuhan, Hubei 430072, China \\ ${ }^{2}$ Laboratory of Nanomaterials and Sensors, School of Physics, Electronics and Communication, Jiangxi Normal University, \\ Nanchang, Jiangxi 330022, China
}

Correspondence should be addressed to Shuangli Ye; slye@whu.edu.cn

Received 29 November 2013; Accepted 23 December 2013; Published 9 January 2014

Academic Editor: Wen Lei

Copyright (C) 2014 Shuangli Ye et al. This is an open access article distributed under the Creative Commons Attribution License, which permits unrestricted use, distribution, and reproduction in any medium, provided the original work is properly cited.

\begin{abstract}
The strain effect on graphene-encapsulated Au nanoparticles is investigated. A finite-element calculation is performed to simulate the strain distribution and morphology of the monolayer and multilayer graphene-encapsulated Au nanoparticles, respectively. It can be found that the inhomogeneous strain and deformation are enhanced with the increasing shrinkage of the graphene shell. Moreover, the strain distribution and deformation are very sensitive to the layer number of the graphene shell. Especially, the inhomogeneous strain at the interface between the graphene shell and encapsulated Au nanoparticles is strongly tuned by the graphene thickness. For the mono- and bilayer graphene-encapsulated Au nanoparticles, the dramatic shape transformation can be observed. However, with increasing the graphene thickness further, there is hardly deformation for the encapsulated Au nanoparticles. These simulated results indicate that the strain and deformation can be designed by the graphene layer thickness, which provides an opportunity to engineer the structure and morphology of the graphene-encapsulated nanoparticles.
\end{abstract}

\section{Introduction}

Metal nanoparticles have extensive technological applications in nanosensors, catalysis, and biomedical engineering [1-3]. The high surface-to-volume ratio, however, makes the naked metal nanoparticles sensitive to the ambient atmosphere and unstable. Therefore, encapsulated metal nanoparticles have attracted increasing attention as an alternative to the naked metal nanoparticles. It has been demonstrated that it is an efficient method to prevent the oxidation of the metal nanoparticles by encapsulating them with graphene shell $[4,5]$. Moreover, the properties of the encapsulated nanoparticles can be significantly modified by the graphene shell due to the unique electronic, optical, and mechanical properties of graphene $[6,7]$. Recently, there is a great interest in the preparation and investigation of the graphene-encapsulated nanoparticles. The high performance of the graphene-encapsulated nanoparticles has a promising potential application [8]. On the other hand, grapheneencapsulated nanoparticles can be compared to the core/shell nanostructures. The properties of this core/shell nanostructure strongly depend on the interplay between the core and shell layer $[9,10]$. Especially, the atomic structure and morphology of the core and shell can be tailored. For example, the graphene shell has been illustrated to be used as compression cell to induce the transformation and reconstruction of the encapsulated nanoparticles by electron or ion beam irradiation, in which the compressive strain plays an important role $[11,12]$. However, because the mechanical property of graphene is very sensitive to the layer number of the graphene shell, the interplay between the core and shell layer can be quite different for the graphene-encapsulated nanoparticles with various shell thickness. Until now, most reports focus on the multilayer graphitic shell with carbon onion structure, which has much weaker mechanical property than that of monolayer graphene. Therefore, information on the graphene thickness-dependent strain distribution may be crucial for designing the atomic structure and morphology of the encapsulated nanoparticles. 


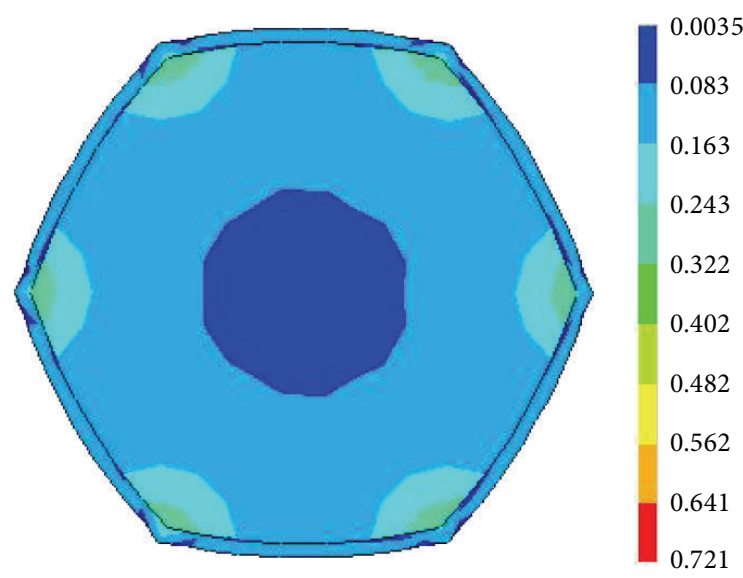

(a)

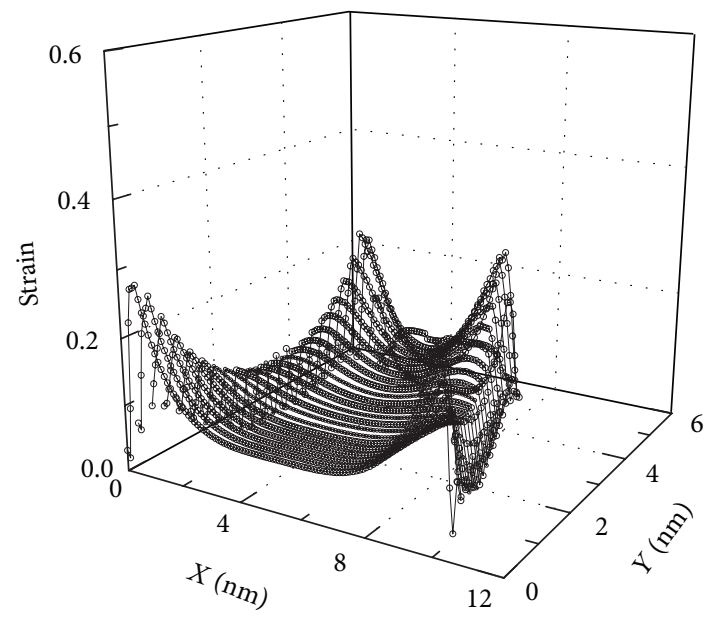

(c)

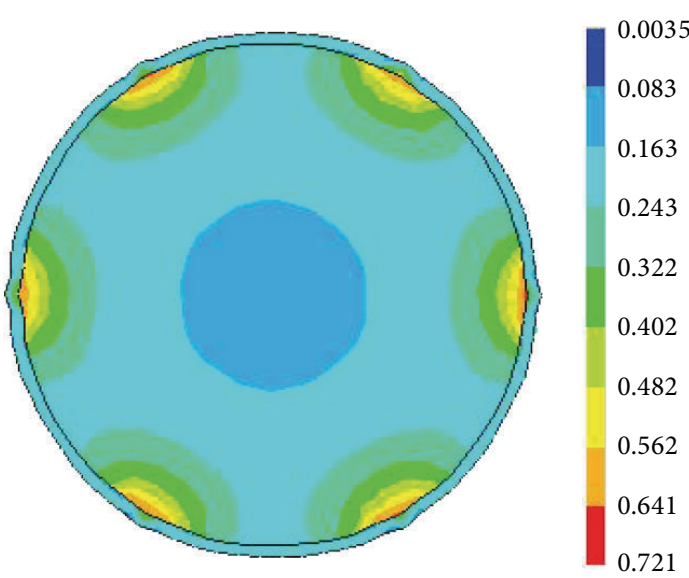

(b)

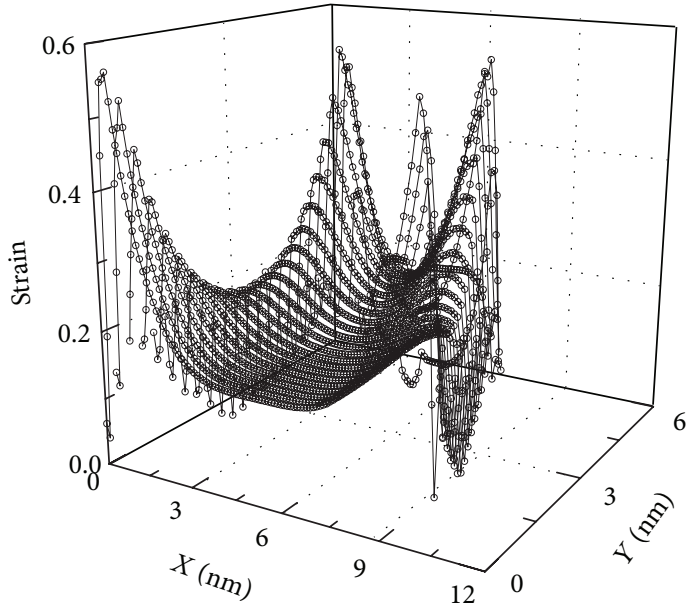

(d)

FIgURE 1: Strain distribution and morphological deformation ((a) and (b)) and $X-Y$ plane strain profiles ((c) and (d)) for the monolayer graphene-encapsulated Au nanoparticle.

In this paper, a finite-element calculation is performed to simulate the strain distribution and deformation of the monolayer and multilayer graphene-encapsulated $\mathrm{Au}$ nanoparticles, respectively. The graphene thicknessdependent deformation of the encapsulated Au nanoparticles can be found. The simulated results reveal that thicknessdependent Young's modulus of the graphene shell is responsible for the tuned strain and deformation. This paper demonstrates that the thickness of the graphene shell can play an important role when the structure and morphology of the encapsulated nanoparticles are modified by the shrinkage of the graphene shell.

\section{Results and Discussions}

In order to investigate the strain tuning effect on the morphology evolution of the nanoparticle, a finite-element (FE) calculation is performed to simulate the dynamic strain distribution of graphene-encapsulated Au nanoparticle with different graphene thickness during the morphology evolution process. FE calculation is a versatile computer simulation technique used for continuum modeling of deformation [13]. The simulations are taken to account for the physical properties of many materials, including elastic anisotropy, thermal expansion, and three-dimensional object shape [14-16]. Moreover, a general qualitative agreement between atomistic strain calculations and continuum elastic models has been demonstrated in nanomaterials $[17,18]$. Recently, using the FE calculation to simulate the strain distribution of nanoparticles has been widely reported $[19,20]$. In our FE model, the Au nanoparticle is considered as hexagonal shape with side length of $5 \mathrm{~nm}$ and the graphene shell encapsulates the nanoparticles without any spare space, which are the initial states. The morphology evolution and the dynamic strain distribution of the graphene-encapsulated Au nanoparticle are simulated with the continuous shrinkage of the graphene shell, which can be experimentally realized by the electron or ion beam irradiation [11, 12]. The thickness of monolayer graphene is $0.335 \mathrm{~nm}$ [21]; the thickness of the bilayer, triplelayer, four-layer, and seven-layer graphene is $0.81,1.285,1.76$, and $3.185 \mathrm{~nm}$ with the layer distance of $0.14 \mathrm{~nm}$, respectively. Young's moduli are 0.8E11, 8.91E11, 3.93E11, 0.51E11, 0.27E11, 


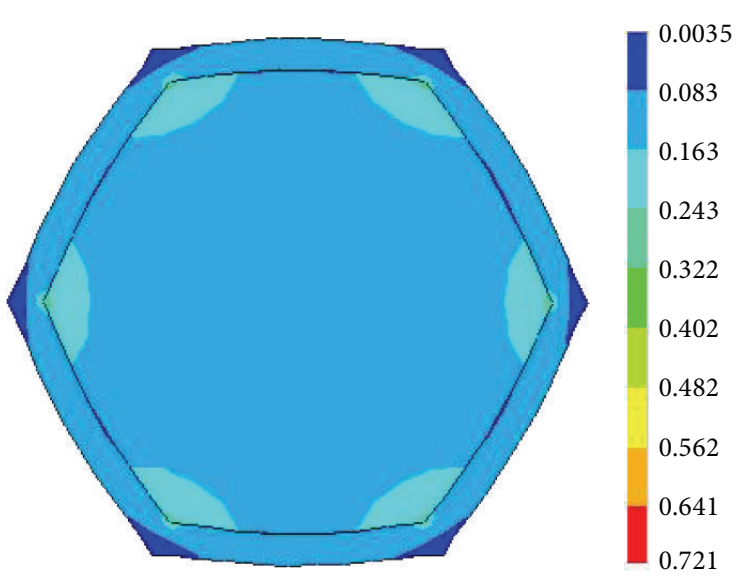

(a)

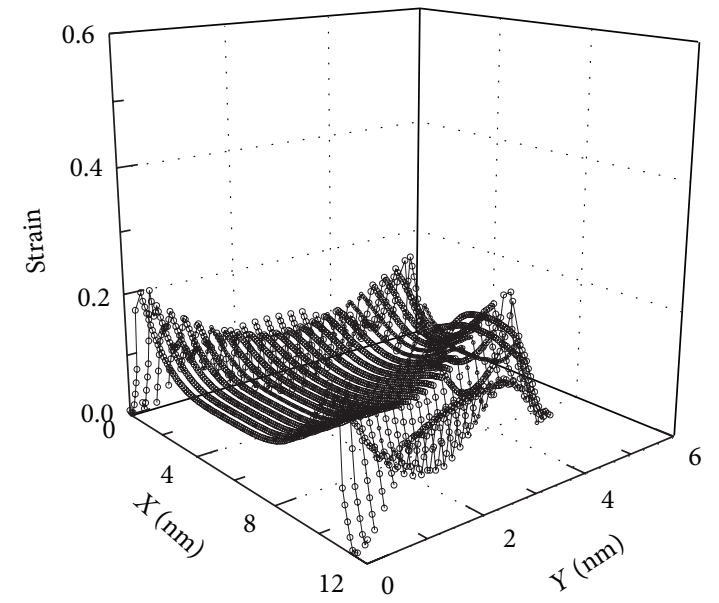

(c)

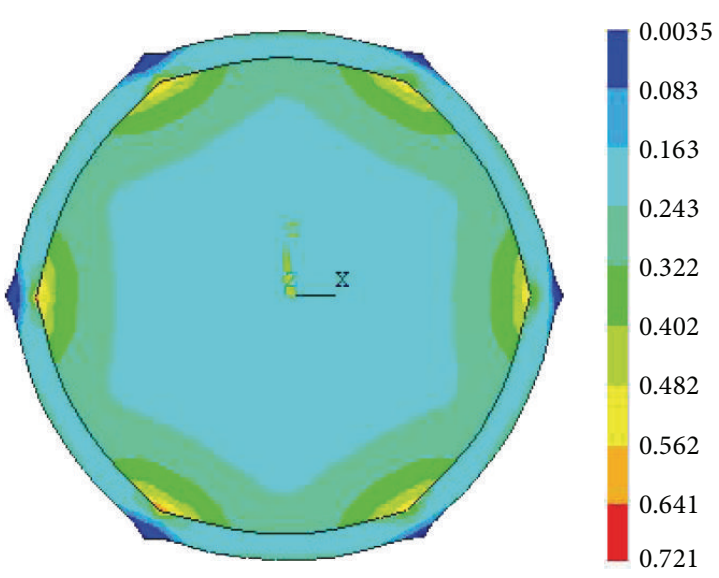

(b)

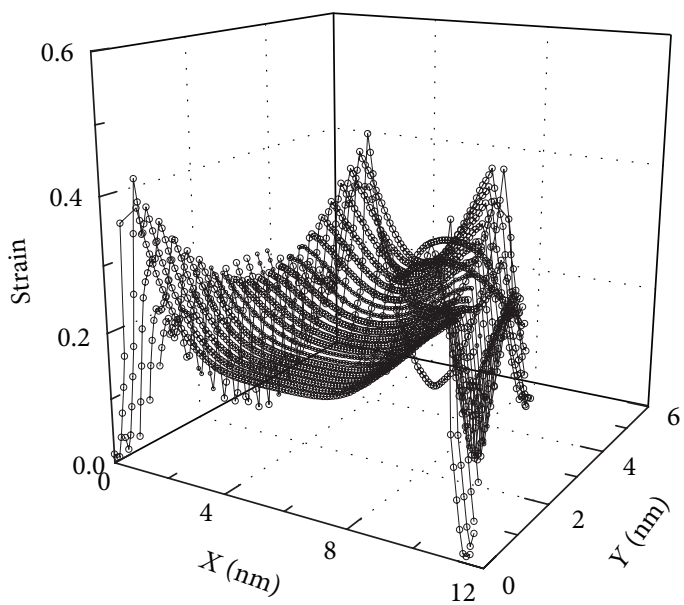

(d)

FigurE 2: Strain distribution and morphological deformation ((a) and (b)) and $X-Y$ plane strain profiles ((c) and (d)) for the bilayer grapheneencapsulated Au nanoparticle.

and $0.27 \mathrm{E} 11 \mathrm{~Pa}[22,23]$ for $\mathrm{Au}$, monolayer, bilayer, triple-layer, four-layer, and seven-layer graphene, while Poisson's ratio is taken to be 0.42 and 0.165 for $\mathrm{Au}$ and graphene, respectively.

Figures 1(a), 1(b), 2(a), and 2(b) show the representative cross-sectional morphologies of the mono- and bilayer graphene-encapsulated $\mathrm{Au}$ nanoparticle during their morphology evolution with the increasing shrinkage of the graphene shell. Correspondingly, Figures 1(c), 1(d), 2(c), and 2(d) illustrate the $X-Y$ plane strain profiles synchronously for these morphologies. As it can be seen in Figures 1(a) and 1(b), the encapsulated $\mathrm{Au}$ nanoparticle in monolayer graphene is transformed from hexagonal to spherical shape with the increasing shrinkage of graphene shell. Figures 1(c) and 1(d) indicate that the $\mathrm{Au}$ nanoparticle is incurred compressive strain by the monolayer graphene shell. Meanwhile, the strain distribution is very inhomogeneous, which increases with the increasing shrinkage of the graphene shell. The compressive strain reaches to the maximum at the six corners of the hexagonal $\mathrm{Au}$ nanoparticle close to the graphene/Au interface. However, the strain decreases very fast in $\mathrm{Au}$ nanoparticle as the position is away from the corners.
Figure 2 shows the morphology evolution of the bilayer graphene-encapsulated Au nanoparticle with the increasing shrinkage of the graphene shell. It can be found that the encapsulated hexagonal Au nanoparticle in bilayer graphene trends to be transformed to spherical shape with the increasing shrinkage of the graphene shell. The Au nanoparticle is incurred inhomogeneous compressive strain by the bilayer graphene shell, however, which is much smaller than that in the monolayer graphene-encapsulated Au nanoparticle, as shown in Figures 2(c) and 2(d). Moreover, compared with the strain distribution in the monolayer graphene-encapsulated $\mathrm{Au}$ nanoparticle, the strain distribution is less inhomogeneous and decreases much smoothly in the encapsulated Au nanoparticle with bilayer graphene as the position is away from the corners. These phenomena can be due to the face that Young's modulus of the bilayer graphene is smaller than that of the monolayer graphene, even though both Young's moduli of the monolayer and bilayer graphene are larger than those of $\mathrm{Au}$ nanoparticle. Therefore, these simulated results indicate that the inhomogeneous compressive strain can be induced with the increasing shrinkage of the graphene shell when Young's modulus of the graphene shell is larger 


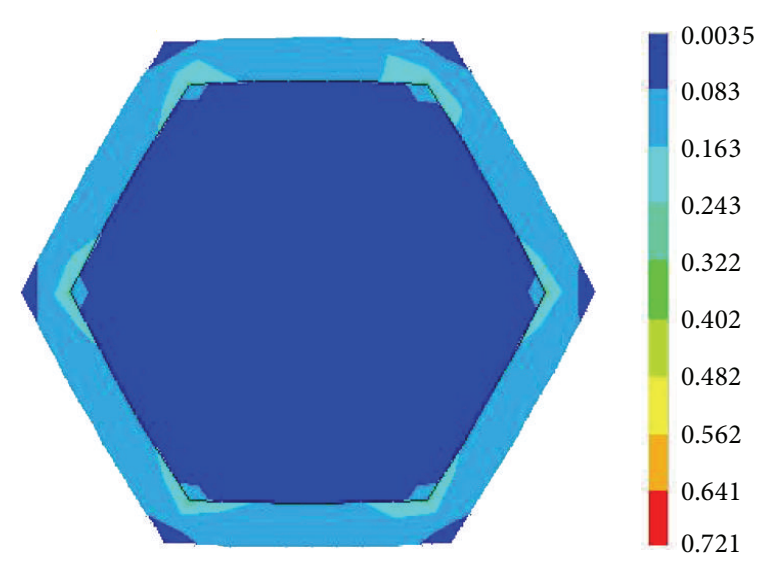

(a)

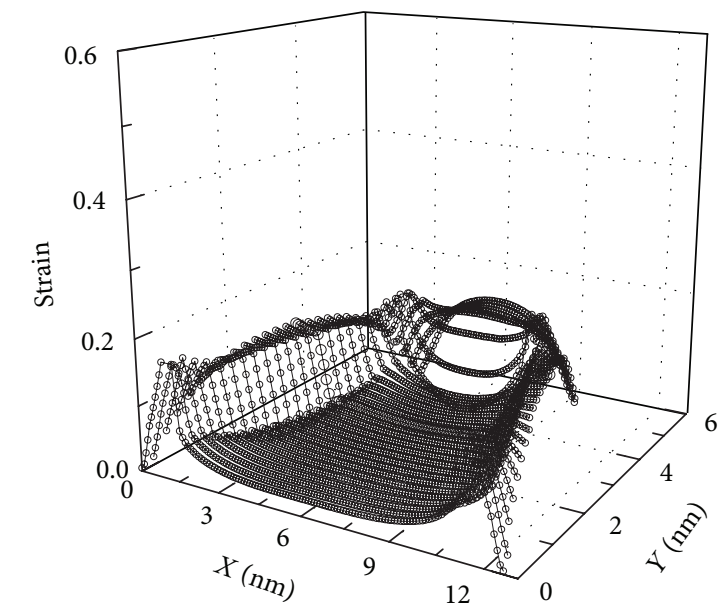

(c)

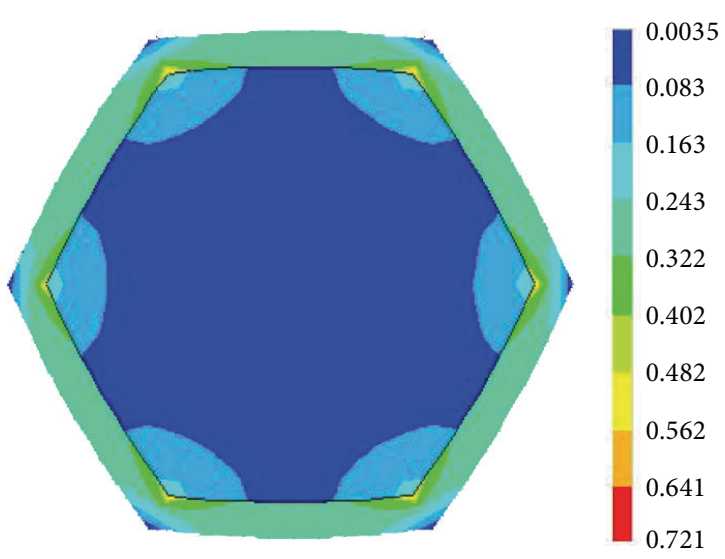

(b)

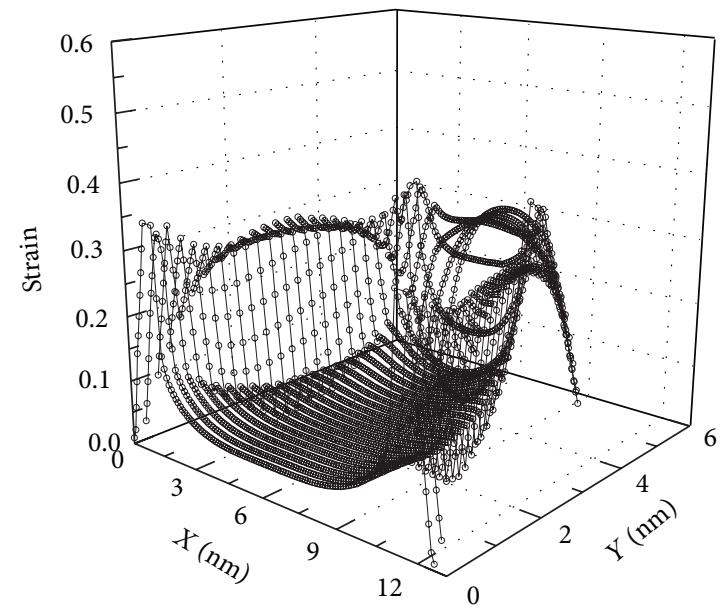

(d)

FIGURE 3: Strain distribution and morphological deformation ((a) and (b)) and $X-Y$ plane strain profiles ((c) and (d)) for the triple-layer graphene-encapsulated Au nanoparticle.

than that of $\mathrm{Au}$ nanoparticle, leading to the deformation of the encapsulated $\mathrm{Au}$ nanoparticle. On the other hand, the strain distribution strongly depends on the graphene thickness which has different Young's modulus, especially at the graphene/Au interface. The thickness-dependent deformation and the strain distribution can be used to tune the structure and the properties of the encapsulated Au nanoparticle.

Figures 3 and 4 are the cross-sectional morphologies and strain distribution for the encapsulated Au nanoparticle with the triple-layer and seven-layer graphene shell with the increasing shrinkage of the graphene shell, respectively. Both Young's moduli of the triple-layer and seven-layer graphene are smaller than those of $\mathrm{Au}$ nanoparticle, because Young's modulus of the graphene decreases with the increasing of the layer number [23]. Compared with that of the monolayer and bilayer grapheme-encapsulated $\mathrm{Au}$ nanoparticle, the simulation results demonstrate that the strongest strain happens in the graphene shell instead of in the Au nanoparticle core for triple-layer and seven-layer grapheme-encapsulated Au nanoparticle, as shown in Figures 3 and 4. The strain in the shrinking graphene shell increases with the increasing of the layer thickness. On the other hand, it can be found that decreasing Young's modulus leads to the decreasing of the inhomogeneous compressive strain on the Au nanoparticle core. Therefore, although the shrinkage of the graphene shell increases, the deformation can be hardly seen for the encapsulated Au nanoparticle with the triple-layer and sevenlayer graphene shell as shown in Figures 3(a), 3(b), 4(a), and 4(b). These simulated results reveal that, with the increasing shrinkage of the graphene shell, the inhomogeneous compressive strain can be enhanced in the graphene layer close to the graphene/Au interface when Young's modulus of the graphene shell is smaller than that of Au nanoparticle. The strong local deformation of the graphene layer at the graphene/Au interface should be observed, which can be used to tune the structure and the properties of the graphene shell. However, the structure of the inner encapsulated $\mathrm{Au}$ nanoparticles is hardly deformed for the thicker graphene shell.

Figure 5 is the comparison of the maximum strain value with different shrinkage of the graphene shell in the graphene-encapsulated Au nanoparticle for monolayer, bilayer, triple-layer, four-layer, and seven-layer graphene 


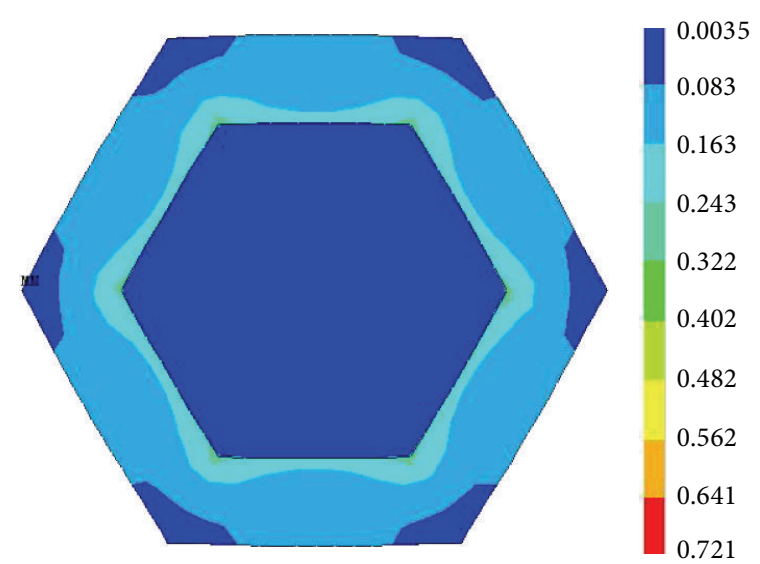

(a)

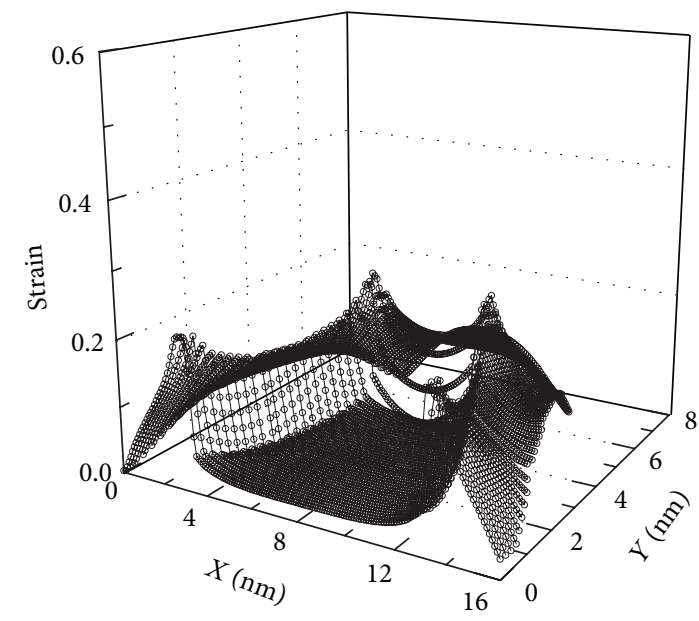

(c)

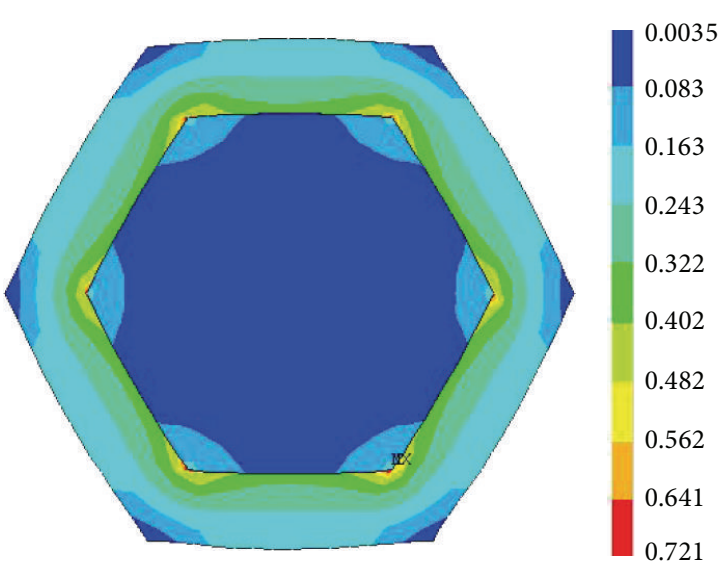

(b)

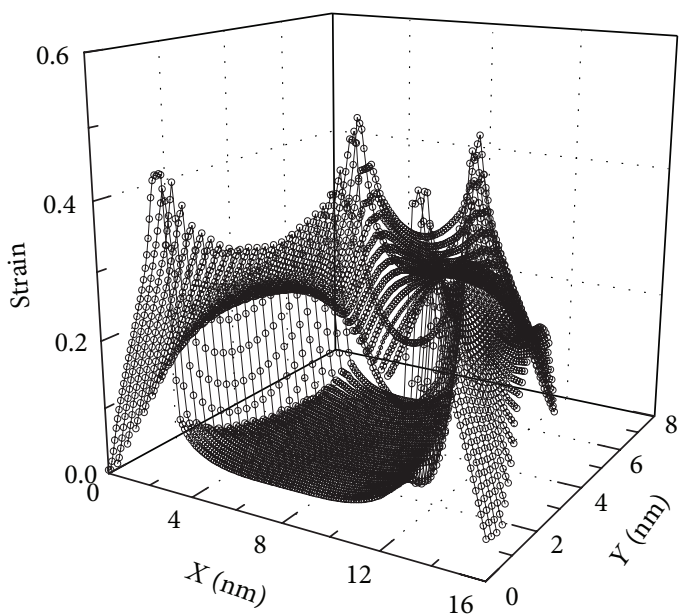

(d)

Figure 4: Strain distribution and morphological deformation ((a) and (b)) and $X-Y$ plane strain profiles ((c) and (d)) for the seven-layer graphene-encapsulated Au nanoparticle.

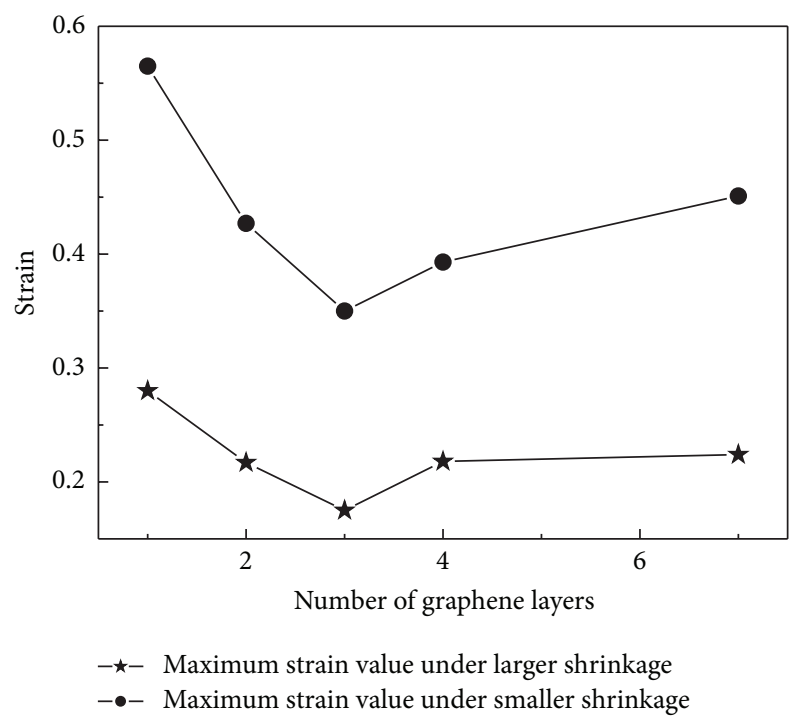

FIGURE 5: The maximum strain values in the graphene-encapsulated Au nanoparticle with different shrinkage of graphene shell. shell, respectively. It can be found that the strongest inhomogeneous strain happens in the monolayer grapheneencapsulated $\mathrm{Au}$ nanoparticle, which can be attributed to highest Young's modulus of the monolayer graphene. The inhomogeneous strain decreases with the increasing graphene thickness and reaches to the smallest value in the triple-layer graphene-encapsulated $\mathrm{Au}$ nanoparticle. With increasing the graphene thickness further, the inhomogeneous strain is enhanced again, which can be due to the enhanced shrinkage by the thicker graphene shell. This result indicates that graphene thickness-dependent Young's modulus is responsible for the tuned strain and deformation of the graphene-encapsulated Au nanoparticle.

\section{Conclusion}

The influence of graphene thickness-dependent Young's modulus on the strain distribution and deformation of the graphene-encapsulated hexagonal Au nanoparticle is investigated by FE simulations. With the shrinkage of the graphene 
shell, it can be found that the encapsulated Au nanoparticle can be deformed when Young's modulus of the graphene shell is larger than that of Au nanoparticle. The compressive strain distributes inhomogeneously in the graphene shell and in the $\mathrm{Au}$ nanoparticle core, especially at the interface. The inhomogeneous strain reaches to the maximum in the monolayer graphene-encapsulated Au nanoparticle. With increasing the number of graphene layer, the inhomogeneous strain on the encapsulated $\mathrm{Au}$ nanoparticles decreases. However, the local strain can be enhanced in the graphene layer close to the graphene/Au interface when Young's modulus of the graphene shell is smaller than that of Au nanoparticle. These simulated results indicate that the strain and deformation of the graphene shell and encapsulated nanoparticles can be designed separately by tuning the graphene layer thickness, which provides an opportunity to engineer the physical properties of the graphene-encapsulated nanoparticles.

\section{Conflict of Interests}

The authors declare that there is no conflict of interests regarding the publication of this paper.

\section{Acknowledgments}

This work is supported by the National Natural Science Foundation of China (Grant nos. 61006080, 11174226, 51371129, 11004087, and 11164008) and the Specialized Research Fund for the Doctor Program of Higher Education no. 20100141120080.

\section{References}

[1] R. Narayanan and M. A. El-Sayed, "Catalysis with transition metal nanoparticles in colloidal solution: nanoparticle shape dependence and stability," Journal of Physical Chemistry B, vol. 109, no. 26, pp. 12663-12676, 2005.

[2] J. Zhao, X. Zhang, C. R. Yonzon, A. J. Haes, and R. P. Van Duyne, "Localized surface plasmon resonance biosensors," Nanomedicine, vol. 1, no. 2, pp. 219-228, 2006.

[3] P. K. Jain, I. H. ElSayed, and M. A. El-Sayed, "Au nanoparticles target cancer," Nano Today, vol. 2, no. 1, pp. 18-29, 2007.

[4] T. Hayashi, S. Hirono, M. Tomita, and S. Umemura, "Magnetic thin films of cobalt nanocrystals encapsulated in graphite-like carbon," Nature, vol. 381, no. 6585, pp. 772-774, 1996.

[5] R. Caudillo, X. Gao, R. Escudero, M. José-Yacaman, and J. B. Goodenough, "Ferromagnetic behavior of carbon nanospheres encapsulating silver nanoparticles," Physical Review B, vol. 74, no. 21, Article ID 214418, 2006.

[6] K. I. Bolotin, K. J. Sikes, Z. Jiang et al., "Ultrahigh electron mobility in suspended graphene," Solid State Communications, vol. 146, no. 9-10, pp. 351-355, 2008.

[7] C. Lee, X. Wei, J. W. Kysar, and J. Hone, "Measurement of the elastic properties and intrinsic strength of monolayer graphene," Science, vol. 321, no. 5887, pp. 385-388, 2008.

[8] S. Yang, X. Feng, S. Ivanovici, and K. Müllen, "Fabrication of graphene-encapsulated oxide nanoparticles: towards highperformance anode materials for lithium storage," Angewandte Chemie-International Edition, vol. 49, no. 45, pp. 8408-8411, 2010.
[9] H. Zeng, S. Sun, J. Li, Z. L. Wang, and J. P. Liu, “Tailoring magnetic properties of core/shell nanoparticles," Applied Physics Letters, vol. 85, no. 5, pp. 792-794, 2004.

[10] A. M. Smith and S. Nie, "Semiconductor nanocrystals: structure, properties, and band gap engineering," Accounts of Chemical Research, vol. 43, no. 2, pp. 190-200, 2010.

[11] A. V. Krasheninnikov and F. Banhart, "Engineering of nanostructured carbon materials with electron or ion beams," Nature Materials, vol. 6, no. 10, pp. 723-733, 2007.

[12] L. Sun, A. V. Krasheninnikov, T. Ahlgren, K. Nordlund, and F. Banhart, "Plastic deformation of single nanometer-sized crystals," Physical Review Letters, vol. 101, no. 15, Article ID 156101, 2008.

[13] K. H. Huebner, D. L. Dewhirst, D. E. Smith, and T. G. Byrom, The Finite Element Method for Engineers, John Wiley \& Sons, New York, NY, USA, 2001.

[14] J. Grönqvist, N. Søndergaard, F. Boxberg, T. Guhr, S. Åberg, and H. Q. Xu, "Strain in semiconductor core-shell nanowires," Journal of Applied Physics, vol. 106, Article ID 053508, 2009.

[15] B. Liu, Y. Huang, H. Jiang, S. Qu, and K. C. Hwang, "The atomicscale finite element method," Computer Methods in Applied Mechanics and Engineering, vol. 193, no. 17-20, pp. 1849-1864, 2004.

[16] H. Hu, L. Onyebueke, and A. Abatan, "Characterizing and modeling mechanical properties of nanocomposites-review and evaluation," Journal of Minerals \& Materials Characterization \& Engineering, vol. 9, no. 4, pp. 275-319, 2010.

[17] D. Barettin, S. Madsen, B. Lassen, and M. Willatzen, "Comparison of wurtzite atomistic and piezoelectric continuum strain models: implications for the electronic band structure," Superlattices and Microstructures, vol. 47, no. 1, pp. 134-138, 2010.

[18] M. Karamehmedovic, R. Schuh, V. Schmidt et al., "Comparison of numerical methods in near-field computation for metallic nanoparticles," Optics Express, vol. 19, no. 9, pp. 8939-8953, 2011.

[19] C. L. Johnson, E. Snoeck, M. Ezcurdia et al., "Effects of elastic anisotropy on strain distributions in decahedral gold nanoparticles," Nature Materials, vol. 7, no. 2, pp. 120-124, 2008.

[20] Z. W. Shan, G. Adesso, A. Cabot et al., "Ultrahigh stress and strain in hierarchically structured hollow nanoparticles," Nature Materials, vol. 7, no. 12, pp. 947-952, 2008.

[21] J. P. Lu, "Elastic properties of carbon nanotubes and nanoropes," Physical Review Letters, vol. 79, no. 7, pp. 1297-1300, 1997.

[22] W. D. Callister Jr., Materials Science and Engineering, John Wiley \& Sons, New York, NY, USA, 3rd edition, 1994.

[23] Y. Zhang and C. Pan, "Measurements of mechanical properties and number of layers of graphene from nano-indentation," Diamond and Related Materials, vol. 24, pp. 1-5, 2012. 

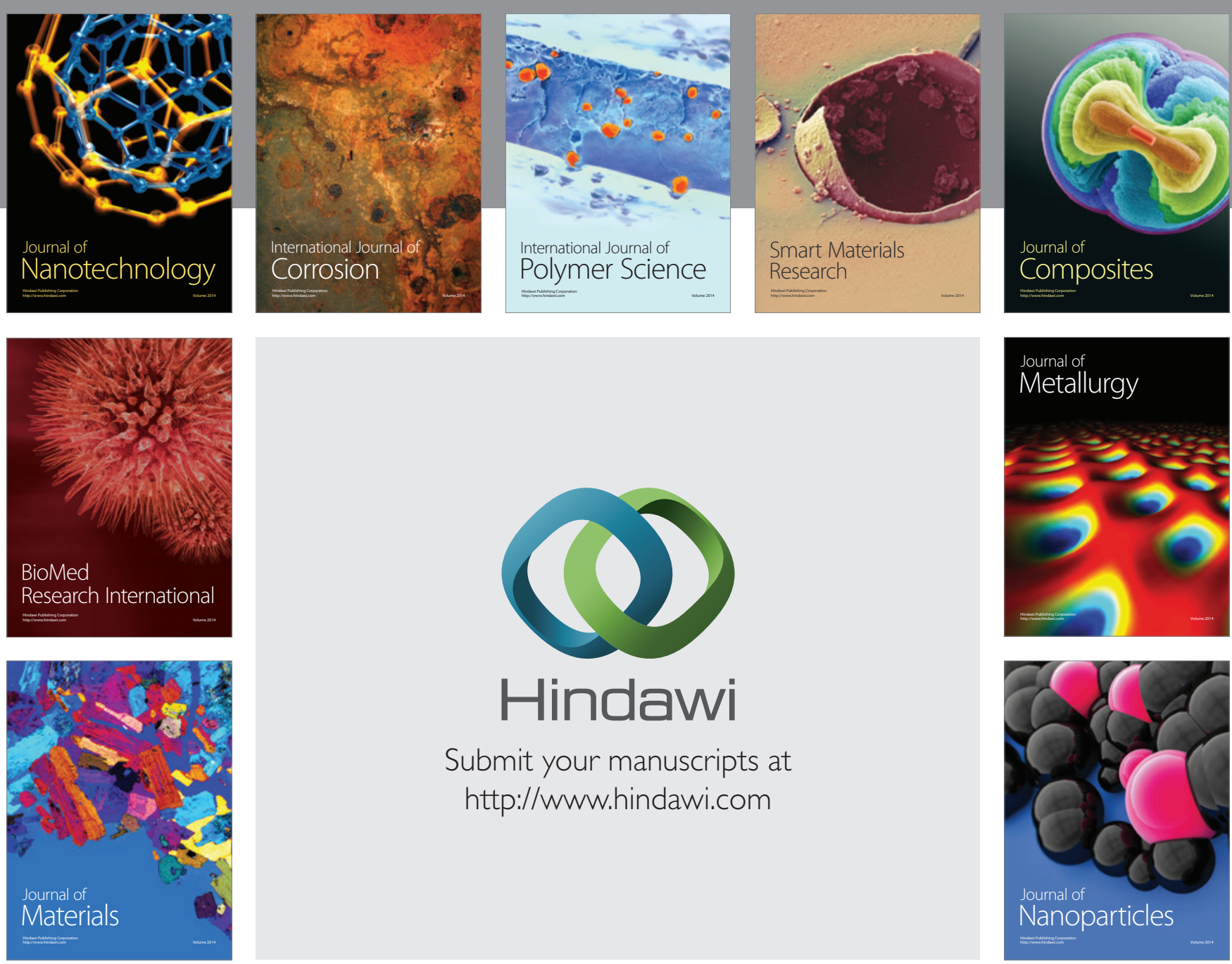

Submit your manuscripts at http://www.hindawi.com
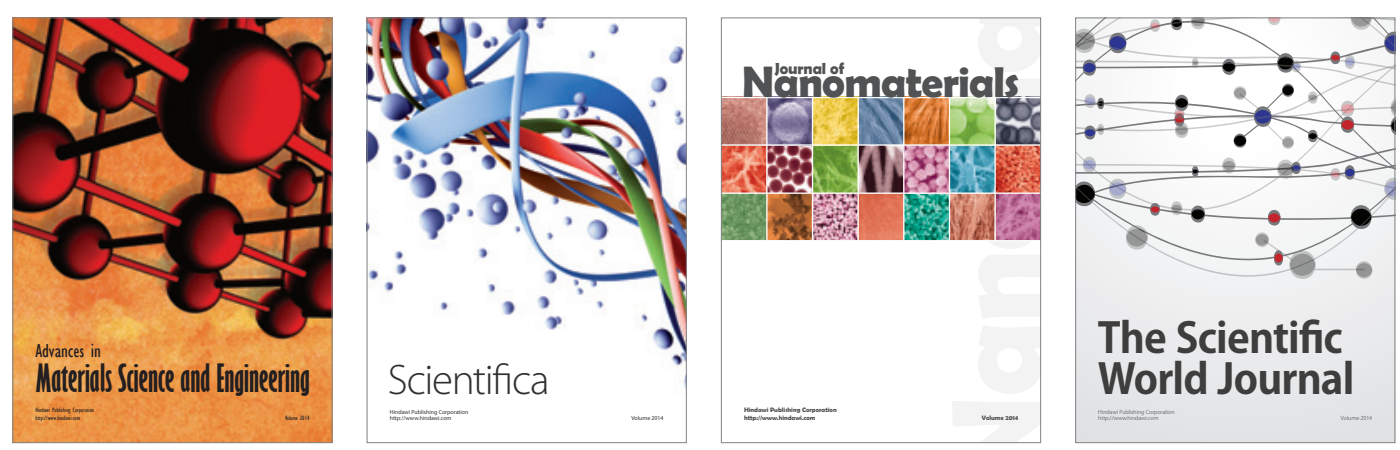

\section{The Scientific World Journal}
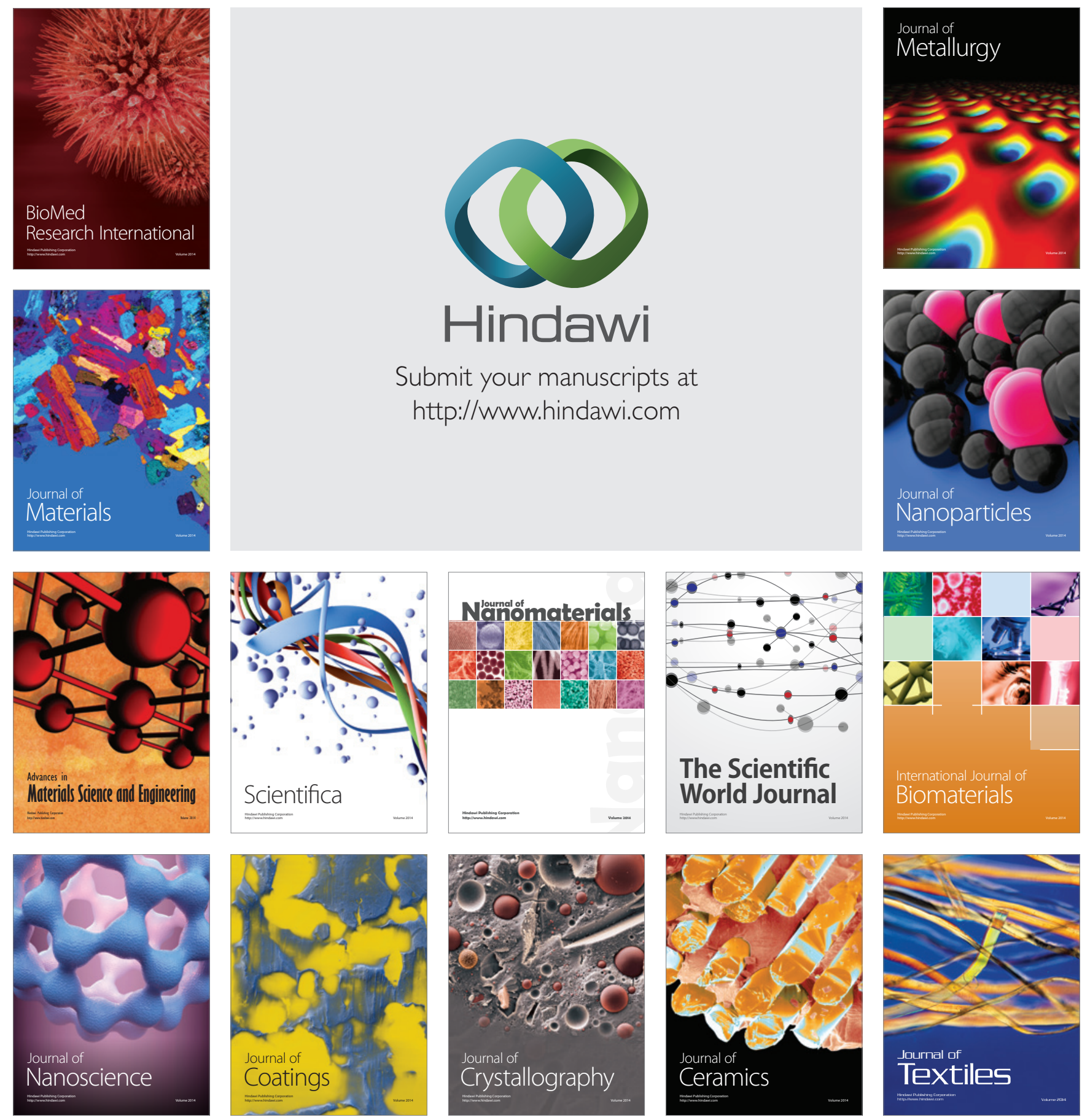